\title{
RECONFIGURING MINIMUM DOMINATING SETS: THE $\gamma$-GRAPH OF A TREE
}

\author{
MiCHELLE EDWARDS \\ Gary MacGillivray ${ }^{1}$ \\ Mathematics and Statistics University of Victoria \\ Victoria, BC, Canada \\ e-mail: edwardsm@uvic.ca \\ gmacgill@uvic.ca \\ AND \\ SHAHLA NASSERASR \\ Mathematics, Nova Southeastern University \\ Ft. Lauderdale, FL, USA \\ e-mail: snasserasr@nova.edu
}

\begin{abstract}
We consider $\gamma$-graphs, which are reconfiguration graphs of the minimum dominating sets of a graph $G$. We answer three open questions about $\gamma$ graphs of trees by providing upper bounds on the maximum degree, the diameter, and the number of minimum dominating sets. The latter gives an upper bound on the order of the $\gamma$-graph.
\end{abstract}

Keywords: domination, reconfiguration.

2010 Mathematics Subject Classification: 05C69.

\section{REFERENCES}

[1] S. Alikhani, D. Fatehi and S. Klavžar, On the structure of dominating graphs (2015). arXiv: 1512.07514

[2] E. Connelly, K.R. Hutson and S.T. Hedetniemi, A note on $\gamma$-graphs, AKCE Int. J. Graphs Comb. 8 (2011) 23-31.

\footnotetext{
${ }^{1}$ Research supported by NSERC.
} 
[3] M. Edwards, Vertex-Criticality and Bicriticality for Independent Domination and Total Domination in Graphs (PhD Thesis, Department of Mathematics and Statistics, University of Victoria, 2015). http://hdl.handle.net/1828/6097

[4] G.H. Fricke, S.M. Hedetniemi, S.T. Hedetniemi and K.R. Hutson, $\gamma$-graphs of graphs, Discuss. Math. Graph Theory 31 (2011) 517-531. doi:10.7151/dmgt.1562

[5] R. Haas and K. Seyffarth, The k-dominating graph, Graphs Combin. 30 (2014) 609-617. doi:10.1007/s00373-013-1302-3

[6] A. Haddadan, T. Ito, A.E. Mouawad, N. Nishimura, H. Ono, A. Suzuki and Y. Tebbal, The complexity of dominating set reconfiguration, Lecture Notes in Comput. Sci. 9214 (2015) 398-409. doi:10.1007/978-3-319-21840-3_33

[7] J. van den Heuvel, The complexity of change, in: S.R. Blackburn, S. Gerke, M. Wildon (Eds.), Surveys in Combinatorics, London Mathematical Society Lecture Notes Series 409 (Cambridge University Press, 2013) 127-160.

[8] T. Ito, E.D. Demaine, N.J.A. Harvey, C.H. Papadimitriou, M. Sideri, R. Uehara and Y. Uno, On the complexity of reconfiguration problems, Theoret. Comput. Sci. 412 (2011) 1054-1065. doi:10.1016/j.tcs.2010.12.005

[9] S.A. Lakshmanan and A. Vijayakumar, The gamma graph of a graph, AKCE Int. J. Graphs Comb. 7 (2010) 53-59.

[10] A. Meir and J.W. Moon, Relations between packing and covering numbers of a tree, Pacific J. Math. 61 (1975) 225-233. doi:10.2140/pjm.1975.61.225

[11] M. Middendorf and F. Pfeiffer, Weakly transitive orientations, Hasse diagrams and string graphs, Discrete Math. 111 (1993) 393-400. doi:10.1016/0012-365X(93)90176-T

[12] A.E. Mouawad, N. Nishimura and A. Suzuki, Reconfiguration of dominating sets (2014) arXiv:1401.5714

[13] N. Sridharan and K. Subramanian, Trees and unicyclic graphs are $\gamma$-graphs, J. Combin. Math. Combin. Comput. 69 (2009) 231-236.

[14] K. Subramanian and N. Sridharan, $\gamma$-graph of a graph, Bull. Kerala Math. Assoc. 5 (2008) 17-34.

Received 27 July 2016 Revised 31 January 2017 Accepted 2 February 2017 